\title{
DNA Methylation Profiles of Immune Cells From Tuberculosis-Exposed Individuals Overlap With BCG-Induced Epigenetic Changes
}

\section{Isabelle Pehrson}

Linköping University

Nina Idh

Linköping University

Clara Braian

Linköping University

Jakob Paues

Linköping University

Jyotirmoy Das

Linköping University

Maria Lerm ( $\nabla$ maria.lerm@liu.se)

Linköping University

\section{Research Article}

Keywords: DNA methylation, tuberculosis, BCG, epigenetics, sputum induction

Posted Date: January 5th, 2022

DOI: https://doi.org/10.21203/rs.3.rs-1157082/v1

License: (9) (i) This work is licensed under a Creative Commons Attribution 4.0 International License.

Read Full License 


\section{Abstract}

The mechanism of protection of the only approved tuberculosis (TB) vaccine, Bacillus Calmette Guérin (BCG) is poorly understood. In recent years, epigenetic modifications induced by BCG have been demonstrated to reflect a state of trained immunity. The concept of trained immunity is now explored as a potential prevention strategy for a variety of infections. Studies on human TB immunity are dominated by those using peripheral blood as surrogate markers for immunity. Here, we instead studied the lung compartment by obtaining induced sputum from subjects included in a TB contact tracing. CD3- and HLA-DR-positive cells were isolated from the collected sputum and DNA methylome analyses performed. Unsupervised cluster analysis revealed that DNA methylomes of cells from TB-exposed individuals and controls appeared as separate clusters, and the numerous genes that were differentially methylated were functionally connected. The enriched pathways were strongly correlated to previously reported epigenetic changes and trained immunity in immune cells exposed to the BCG vaccine in human and animal studies. We further demonstrated that similar pathways were epigenetically modified in human macrophages trained with BCG in vitro. Altogether, our study demonstrates that similar epigenetic changes are induced by M. tuberculosis and BCG.

\section{Introduction}

Tuberculosis (TB) is a pulmonary infection of pandemic rank and an expansion of the current toolkit for diagnosis, prevention and treatment is critical for reaching the United Nations' Sustainable Development Goals for 2030 of ending the TB epidemic ${ }^{1}$. TB is caused by Mycobacterium tuberculosis, which transmit via aerosols and target alveolar macrophages in exposed individuals ${ }^{2}$. In susceptible hosts, the bacteria replicate inside the macrophages and use them as trojan horses in order to disseminate in the tissues ${ }^{3}$. Bacillus Calmette Guérin, a non-virulent derivative of Mycobacterium bovis, has been used for almost a century as a vaccine against TB, with variable efficacy. Numerous studies have failed to provide correlates of protection, leaving the vaccine mechanism elusive. In recent years, the concept of trained immunity has evolved as an epigenetically encoded immune memory that can be triggered by a variety of stimuli and is reflected in a reprogrammed immune state characterized by a higher magnitude of response to subsequent pathogen challenges. The discovery of epigenetically regulated antimicrobial defense mechanisms goes beyond the classical understanding of immune defense and opens up a new field of research. Along this line, we have demonstrated that administration of the BCG vaccine to healthy subjects induced profound epigenetic alterations in immune cells, which correlated with enhanced antimycobacterial activity in macrophages isolated from the vaccinees ${ }^{4}$. The changes were reflected in the DNA methylome, with the strongest response being recorded within weeks after vaccination ${ }^{4}$. Our observation that BCG induces alterations of the DNA methylome of immune cells has later been confirmed by others ${ }^{5,6}$. Since BCG vaccination reflects an in vivo interaction between immune cells and viable mycobacteria, we here hypothesized that natural exposure to $M$. tuberculosis would induce similar changes not only in TB patients, but also in individuals who have been exposed to TB. Analyses of DNA methylomes of immune cells isolated from lungs and peripheral blood allowed us to identify distinct DNA 
methylation (DNAm) signatures in TB-exposed individuals. Pathway analyses revealed strong overlaps with previous studies on BCG-induced epigenetic signatures that could be correlated with protection against $M$. tuberculosis. We therefore set up an experiment to scrutinize the anti-mycobacterial mechanisms correlating with BCG-induced epigenetic reprogramming in human primary macrophages. BCG-trained macrophages were subjected to DNA methylome analysis and in parallel monitored in a live cell imaging system during exposure to $M$. tuberculosis. Again, the DNAm change in vitro highly overlapped with the signatures of the TB-exposed individuals and the previously identified BCG-induced epigenetic changes.

\section{Results}

\section{Study design}

To determine epigenetic changes in the immune cells in TB-exposed individuals, we recruited subjects enrolled in a TB contact tracing at Linköping University Hospital, Sweden, according to standard contact tracing routines (household contacts and persons with $>8$ hours of interaction with the index case). First, we included one index case and three contact tracing subjects who were ethnicity-matched and not relatives (Table 1). The patient with active tuberculosis (referred to as 'index case') was diagnosed with drug-sensitive pulmonary TB and had completed two out of six months of standard treatment at the time of sample collection. Age- and ethnicity-matched individuals were included as controls (Table 1). In the second part of the study, we included three subjects (one TB patient and two subjects in other contact tracings) who were not ethnicity-matched (Table 1). All included subjects except one (a TB contact) had been BCG-vaccinated at least two years before participation (Table 1). Interferon-Gamma Release Assay (IGRA) status was determined and among the exposed individuals, two were positive (including the index case) and among the controls, one individual (C2) was classified as 'borderline'- positive ${ }^{7}$ (Table 1). From induced sputum, we used an established protocol for the separate isolation of HLA-DR-positive and CD3 positive cells ${ }^{8}$, whereas the PBMC fraction extracted from blood was kept as a mixed population. For the in vitro experiment with BCG and $M$. tuberculosis exposure, human primary monocytes were collected from blood samples from healthy blood donors and differentiated into macrophages (Fig. 1).

\section{DNA methylome data from TB-exposed individuals form a separate cluster}

DNA isolation from the studied cell populations was followed by global DNAm analysis using the Illumina $450 \mathrm{~K}$ protocol. After curation of the data ${ }^{9}$, the datasets were subjected to unsupervised hierarchical cluster analysis based on DNA CpG methylation $\beta$-values. This unsupervised approach accurately clustered the participants into TB-exposed and controls based on the DNA methylome data derived from both HLA-DR- and CD3-positive cell populations (Fig. 2a-b). On the other hand, in the PBMCderived dataset, the TB index case appeared outside the clusters and two of the controls clustered with the other exposed individuals, one of them ("Con_2") being the individual identified as IGRA borderlinepositive (Fig. 2c). 
Next, we identified the differentially methylated $\mathrm{CpG}$ sites (DMCs) and differentially methylated genes (DMGs) by comparing the TB-exposed and control groups for each cell population. To filter out the most significantly altered DMGs in the dataset, the stringency criteria of $\log _{2}>|0.3|$ fold increased or decreased $\beta$-values and Benjamini-Hochberg (BH)-corrected $p$-value $<0.05$ (HLA-DR), $<0.1$ (CD3) and $<0.2$ (PBMC) were applied. The results are depicted as volcano plots, which show that DNA methylomes of TB-exposed most strongly differ in the HLA-DR cells as compared to control subjects, followed by the CD3 population, whereas PBMC datasets reveled fewer DMGs (Fig. 2d-f, Suppl. Table 1). To highlight the locus position of the DMGs, chromosome maps were constructed (Suppl. Fig. S1a-c). Using the same stringency criteria as for the HLA-DR analysis, we tested whether DMGs would emerge when the datasets were arranged in other possible groups as derived from the demographics ( $>/<$ median age, sex, IGRA status). Neither age nor IGRA status generated any significant DMGs with these settings, and gender rendered three DMGs (X and $Y$ chromosomes were removed in the initial filtering prior the analysis) in the HLA-DR positive and in the CD3 positive cells (Suppl. Table 1).

\section{Functional enrichment analysis reveals common and unique interactomes in the datasets}

Using the PANTHER Database, we investigated whether the identified DMGs were enriched in known pathways (Fig. 3a-c). The analysis revealed enrichment in pathways with relevance for TB infection, including hypoxia-inducible factor (HIF)1-a activation, Vitamin D metabolism and p38, Wnt, Notch, interleukin, chemokine, and cytokine signaling pathways ${ }^{10-17}$. Common pathways shared between at least two of the cell populations included B cell activation, glycolysis, angiotensin II signaling, and cholecystokinin signaling. Notably, several pathways named after their known functions in the nervous system were enriched in the studied cell populations, including pathways involved in axon guidance and adrenaline, acetylcholine and glutamate signaling. In the PBMC population but not in the lung cell populations, the interferon-y signaling pathway was identified among the enriched pathways.

Comparisons across cell populations and species reveals the existence of a common DNA methylomebased biosignature in mycobacteria-exposed immune cells

Given the fact that the interaction between mycobacteria and eukaryotes is evolutionary ancient, we predicted that highly conserved pathways exist that are common among the studied cell populations. Comparing the identified DMGs from the HLA-DR, CD3 and PBMCs in a Venn analysis, we discovered 185 common DMGs (Fig. 4a). We expanded the Venn analyses to include data from our previous work on $B C G$ vaccine-induced DMGs that correlated with enhanced mycobacterial control ${ }^{4}$, as natural exposure to TB and BCG vaccination both represent in vivo encounters between mycobacteria and host immune cells. Even though the routes of mycobacterial exposure differ profoundly in these settings, a set of 151 DMGs could be identified as overlapping between our previous BCG study and all cell populations studied here (Fig. 4b), suggesting that a highly conserved epigenetic response to mycobacterial challenge exists. 
In 2018, Hasso-Agopsowicz et al. described alterations in DNAm patterns in PBMCs from BCG-vaccinated individuals, with concomitant enrichment in many immune-related pathways ${ }^{5}$. In order to compare that study with ours, we performed PANTHER analysis with the 185 common DMGs and matched the identified enriched pathways, revealing that $75 \%$ of those pathways were the same as in the present study (Fig. 5a), further corroborating the relationship of the altered DNAm patterns induced through TB exposure and BCG-induced changes. In a study by Kaufmann et al., BCG-induced alterations of the epigenome in mice was correlated with protection against $M$. tuberculosis infection ${ }^{18}$. In order to translate our human DNA methylome signature to the signature identified in the mouse study, we searched for pathway overlaps between the two using Gene Ontology (GO) enrichment analysis (Suppl. Fig. S2a-c). Figure 5b demonstrates that for our PBMC data, the GO terms "biological processes" overlapped $100 \%$ with the mouse study (same cell population) and to $31 \%$ and $65 \%$ for HLA-DR- and CD3positive cells, respectively. In 2014, Saeed et a/ ${ }^{19}$ demonstrated the induction of trained immunity pathways by another immune-training agent, $\beta$-glucan. We assessed possible pathway overlap with that study and although there were fewer overlaps as compared to the BCG-induced pathways described above. Again, the strongest correlation was found in the PBMC fraction, in this case in the GO terms "cellular components" (Fig. 5c).

To validate how well the $284 \mathrm{CpG}$ sites corresponding to the 185 overlapping DMGs performed in an unsupervised cluster analysis, we included one additional TB patient and two contacts, and collected HLA-DR-positive cells from induced sputum, as the DNA methylome data from this cell type was clearly outperforming the others with respect to accurate separation of the groups. Figure 6 shows a $k$ meansbased dendrogram with a heatmap of the $\beta$ values of the $284 \mathrm{CpG}$ sites from both previous and new subjects' DNAm data, revealing a distinct separation of the subjects in accordance with TB exposure.

\section{In vitro BCG training of macrophages induced DNAm changes corresponding to exposure to TB}

To investigate whether the BCG-induced epigenetic changes can be mimicked in vitro, we set up a BCG training experiment with macrophages isolated from donor blood that were trained with BCG and subsequently infected with the virulent $M$. tuberculosis strain H37Rv (expressing GFP) and monitored during the course of infection. In a subset of donors, BCG-trained cells displayed an increased capacity to kill M. tuberculosis (Fig. 7a-b) while cell viability was retained (Fig. 7c-d). DNAm analyses was performed on DNA isolated from these donors' macrophages 24 hours after BCG training. We identified 7471 DMGs with the stringency criteria of DNAm difference $>30 \%$ and BH corrected $p$-value $<0.01$. A PANTHER pathway analysis based on the identified DMGs showed significant enrichment in the Wnt signaling pathway, cadherin signaling pathways and angiogenesis, overlapping with the over-represented pathways in the HLA-DR+ and CD3+ cells from the TB-exposed individuals (Fig. 7e).

\section{Discussion}


In this study, we present data suggesting that exposure to TB generates a distinct DNAm signature in pulmonary immune cells. The signature was found not only in those with active or latent TB infection, but also in individuals who are exposed but IGRA-negative. The finding that healthy, TB-exposed individuals also carry the signature opens the possibility that the epigenetic alterations reflect a host-beneficial reprogramming of the immune mechanisms rather than being induced by $M$. tuberculosis as a step to evade the immune defense. This notion is supported by our observation that the DMGs identified in the present study strongly overlapped with the epigenetic alterations identified in the in vitro BCG-trained macrophages, and the previously reported DNAm changes induced during BCG vaccination, which correlated with increased anti-mycobacterial capacity of macrophages ${ }^{4}$. In addition, we demonstrate that the GO data derived from our dataset display a strong overlap with data from a study on protective BCG vaccination in mice ${ }^{6}$.

BCG vaccination has convincingly been shown to induce heterologous immunity protecting against childhood mortality from other causes than $\mathrm{TB}^{20,21}$. Based on our finding that natural TB exposure and BCG vaccination trigger similar epigenetic changes we propose the hypothesis that a "beneficial exposure" to TB exists, which protects against other infections through heterologous immunity. Along the same line, it has been shown that a substantial fraction of individuals exposed to TB can be defined as 'early clearers', since they remain tuberculin skin test or IGRA negative ${ }^{22}$, suggesting effective eradication of the infection ${ }^{22}$. Identifying these early clearers and understanding the biology behind their resistance to TB infection could move the field forward towards novel strategies of TB prevention.

In concordance with the fact that macrophages constitute the main niche for mycobacterial replication, the strongest enrichment of DNAm changes was observed in the HLA-DR-positive cell population, which is dominated by alveolar macrophages. The pathways identified to be enriched in the HLA-DR-positive population have been described in the context of trained immunity, BCG exposure and TB. For example, activation of Hypoxia-Inducible Factor $1 \mathrm{a}$ and glycolysis pathways (P00030 and P00024, respectively) are hallmarks of macrophages that have undergone the epigenetic changes reflective of trained immunity (reviewed in ${ }^{23,24}$ ), which is induced in myeloid cells upon BCG-stimulation ${ }^{22,23}$. VEGF-release (P00056) by macrophages has been shown to recruit immune cells during granuloma formation ${ }^{25}$. Further, vitamin D has been shown to strengthen the anti-mycobacterial activity of macrophages ${ }^{11,26}$, and upregulation of components of the vitamin D pathway is linked to the production of anti-microbial peptides ${ }^{12}$, providing a possible effector mechanism for mycobacterial control. Recent literature on immune regulation through $T$ cell-derived acetylcholine ${ }^{27,28}$ attributes relevance to the acetylcholine receptor pathway identified among the HLA-DR pathways.

Although macrophages and lymphocytes are not generally viewed as having many similarities, we found 34 of the identified pathways to overlap between HLA-DR- and CD3-positive cells. In data derived from the CD3 and PBMC populations, both of which represent lymphocytes, overlaps were identified for glycolysis, glutamate receptor and angiotensin II pathways. Interestingly, a metabolic shift towards increased glycolysis, representative of the Warburg effect, has been strongly associated with trained immunity ${ }^{23}$. 
However, the literature is dominated by the view that this event takes place in trained myeloid cells, while we identified this circuit in CD3 cells (T lymphocytes) and not in the HLA-DR cells (mainly macrophages). The glutamate receptor is widely expressed on immune cells and has been described as having an important regulatory role in $T$ cells, which can also produce and release glutamate ${ }^{29}$. The role for angiotensin II pathway in TB remains elusive, while Angiotensin II Converting Enzyme 2 has been in the spotlight due to fact that the SARS-CoV2 virus utilizes it as a receptor for entry into host cells ${ }^{30}$. In the PBMC population, which over all showed a weaker epigenetic response, we found the interferon- $\gamma$ signaling pathway, which has a well-established role in anti-mycobacterial defense (reviewed in ${ }^{31}$ ), among the reprogramed pathways. Finally, several studies have ascribed Wnt pathways

immunomodulating functions and induction during $M$. tuberculosis infection (reviewed in ${ }^{14}$ ) and $\mathrm{M} 1$ and M2 macrophages express distinct patterns of Wnt ligands.

Taken together, we present data supportive of mycobacteria exposure-induced DNAm changes that correlate with previous findings from studies on BCG vaccination including TB protection and trained immunity.

\section{Materials And Methods}

\section{Study design and ethics}

Patients with pulmonary TB, healthy participants with a history of TB-exposure and healthy controls, with an age ranging from 18 to 53 years, were enrolled at Linköping University Hospital and Linköping University, respectively. Included subjects (Table 1) donated peripheral blood and induced sputum samples ${ }^{8}$ following oral and written informed consent (ethical approval obtained from the Regional Ethics Review Board (EPN) in Linköping, no. 2016/237-31). The study protocol included questionnaires on respiratory and overall health, the evaluation of IGRA-status and sputum samples for DNA extraction. The subjects' samples and questionnaires were not linked to any personal information at any stage of the study. The sputum sample collections were performed in accordance with guidelines from the Department of Respiratory Medicine at Linköping University Hospital. The IGRA samples (QuantiFERONTB Gold) were collected and analyzed by medical personnel according to the guidelines at the Department of Clinical Microbiology at Linköping University Hospital. For in vitro experiments, deidentified buffy coats were purchased from the blood facility at Linköping University Hospital. The buffy coats were obtained from healthy blood donors, who gave written consent of research use for the blood products.

\section{Induced sputum and pulmonary immune cell isolation}

Induced sputum is a well-tolerated, non-invasive method to collect cells from the surface of the bronchial airways after inhalation of a hypertonic saline solution. The procedure of sputum induction takes approximately 30 minutes and is both cost effective and safe with minimal clinical risks ${ }^{32}$. Sputum specimens were collected as described by Alexis et $a^{\beta 3}$, with the following modifications: premedication 
with an adrenergic $\beta 2$-agonist, salbutamol (Ventoline, $1 \mathrm{ml} 1 \mathrm{mg} / \mathrm{ml}$ ) was administrated before the inhalation of hypertonic saline, using a nebulizer (eFlow, PARI). The subsequent steps of sputum processing were adopted from Alexis et al. (2005) $)^{34}$ and Sikkeland et $a^{\beta 5}$. The HLA-DR and CD3-positive cells were isolated using superparamagnetic beads coupled with anti-human CD3 and Pan Mouse IgG antibodies and HLA-DR/human MHC class II antibodies (Invitrogen Dynabeads, ThermoFisher, cat no. 11041 and 14-9956-82, respectively). An initial positive selection was done with CD3 beads followed by a positive HLA-DR selection. Bead-coating and cell isolation were performed according to manufacturer's protocol.

\section{PBMC isolation}

PBMCs were isolated from whole blood (TB-exposed and controls) or from leukocyte rich fractions of blood obtained from healthy volunteers (Linköping University Hospital blood bank, Linköping). Isolation was performed by the method of density gradient centrifugation using Lymphoprep (Axis-Shield) and Sepmate-50 tubes (Stemcell Technologies) according to manufacturer's protocol. IGRA status was determined on the whole-blood samples using QuantiFERON-TB Gold (Cellestis) following the manufacturer's instructions.

\section{Cell culture and in vitro BCG training}

Following PBMC isolation cells were seeded in cell culture treated flasks in Dulbecco's Modified Eagle's Medium (DMEM, Invitrogen) with $25 \mathrm{mM}$ hepes, $100 \mathrm{U} / \mathrm{ml}$ penicillin and $100 \mu \mathrm{g} / \mathrm{ml}$ streptomycin (PEST, Gibco). Cells were incubated in $37^{\circ} \mathrm{C}$ for $2 \mathrm{~h}$ before the non-adherent lymphocytes were washed away using warm Krebs-Ringer Glucose buffer (made in house). Complete DMEM supplemented with 10\% pooled human serum, $2 \mathrm{mM} \mathrm{L-glutamine,} 100 \mathrm{U} / \mathrm{ml}$ penicillin and $100 \mu \mathrm{g} / \mathrm{ml}$ streptomycin (all from Gibco) was then added to the cells along with immune training agents, or medium only as negative control for $24 \mathrm{~h}$. For the DNA methylation analysis and data presented in figure 7a-d we used $10 \mu \mathrm{g} / \mathrm{ml}$ of BCG (freeze dried $M$. bovis bacillus Calmette-Guérin Danish Strain). Training agents were washed off and the cells were incubated for 6-7 days in complete medium with media change every 2-3 days. The cells were washed with PBS followed by trypsinization and reseeding of 5000 cells/ well in a 384-well plate (Corning Falcon) for infection experiments. DNAm analysis DNA was isolated at day 6 after training with BCG.

\section{DNAm sequencing and data analysis}

DNA from PBMCs, HLA-DR-positive, CD3-positive cells, and the cultured human primary macrophages was extracted using the AllPrep DNA/RNA Mini Kit (Qiagen, Hilden, Germany) according to the manufacturer's instructions. Genome-wide DNAm analysis was performed using the HumanMethylation450K BeadChip (Illumina, USA) array (for the HLA-DR, CD3, PBMC samples) and reduced representation bisulfite sequencing (Diagenode's RRBS) read in Illumina's HiSeq 2000 (for the cultured human primary macrophages) at the Bioinformatics and Expression Analysis (BEA) Core Facility at Karolinska Institute, Stockholm. The methylation profiles from the HumanMethylation450K BeadChip analysis for each cell type were analyzed from the raw IDAT files in R (v4.0.2) using the minfi (v1.36.0) with subset-quantile within array (SWAN) normalization ${ }^{36,37}$ and $\operatorname{ChAMP}(\mathrm{v} 2.19 .3)$ with beta-mixture 
quantile normalization (BMIQ) packages ${ }^{38,39}$. The type I and type II probes were normalized using the quantile normalization method. Using the default setup of the ChAMP package, following probes were filtered out: i) probes below the detection $p$-value ( $>0.01)$, ii) non-CpG probes, iii) multi-hit probes, and iv) all probes of $X$ and $Y$ chromosomes. Cell type heterogeneity was corrected for the PBMC cell types using the Houseman algorithm ${ }^{40}$ and batch effects were fixed using ComBat from the SVA package (v3.38.0). Differential methylation analysis was performed with the linear modeling (ImFit) using the limma package $^{41}$ (v3.46.0) in a contrast matrix of the TB-exposed and TB-non-exposed (Control) individuals. All Differentially methylated CpGs (DMCs) were considered significant at the Bonferroni-Hochberg (BH) corrected $p$-value $<0.05$ (for HLA-DR cell types), $<0.1$ (for CD3 cell types) and $<0.2$ (for PBMC cell types). The DNA from the human primary macrophages was sequenced using the Diagenode's RRBS due to a lower DNA yield. The sequenced reads were quality checked using the FastQC ${ }^{42}$ (v0.11.9). The sequences were trimmed to remove artificially filled-in cytosines at the 3 ' end using the TrimGalore (v.0.6.5)( https://github.com/FelixKrueger/TrimGalore) with a phred score cutoff of 20 and quality checked again after trimming. The trimmed sequences were aligned with the human reference genome (hg38.13) using Bowtie $2^{43}$ and removed the duplicates using the Bismark (v.0.22.3) ${ }^{44}$. The methylation extractor from Bismark was used to extract the $\mathrm{CpG}$ methylation data from the sequences. The SAMtools (v1.7) package ${ }^{45}$ was used to sort the binary alignment files (BAM) files on $\mathrm{CpG}$-site chromosomal location and converted to sequence alignment map (SAM) files. The methylated and unmethylated $\mathrm{CpG}$ counts were extracted and combined using the DMRfinder ${ }^{46}$ (v0.3) package in R (v4.0.2). The CpG-sites located in the $X$ and $Y$ chromosome as well as CpG-sites from mitochondrial DNA were filtered out. For differential methylation analysis the methylKit ${ }^{47}(\mathrm{v} 1.18 .0)$ package was used. CpG-sites with a read coverage $>10$ with both methylated and unmethylated reads were removed from the analysis. The function "calculateDiffMeth" from methylKit package using logistic regression was used as statistical test with "before" and "after" BCG-training as treatment used to identify differentially methylated CpG-sites DMCs. The DMCs were annotated to the official gene symbol using org.Hs.eg.db (v3.12) and AnnotationDbi (v1.52) packages using the human genome version hg38. DMGs were defined as sited with a methylation difference of $30 \%$ between the conditions and an $\mathrm{BH}$ corrected $p$-value of $<0.01$.

\section{Unsupervised cluster analysis}

Hierarchical clustering of the all TB-exposed and control individuals was performed with the normalized $\beta$-values obtained after the data filtration in each cell type individually. The distance was calculated using

the Euclidean distance matrix. The dendextend $d^{48}(\mathrm{v} 1.14 .0)$ and $a p e^{49}(\mathrm{v} 5.4-1)$ packages in $\mathrm{R}$ were used to construct the horizontal hierarchical plots from the three different cell populations using the hclust and dendrogram functions.

\section{Structural annotations}

The EnhancedVolcano package ${ }^{50}$ (v1.8.0) was used to generate the individual volcano plots from all cell populations. The ChromoMap package ${ }^{51}$ (v0.3) was used to annotate and visualize the genome-wide 
chromosomal distribution of the DMGs. The interactive plots were generated using the plotly (v4.9.3) package.

The heatmaps were generated from the filtered DMGs with their respective CpGs for each cell type using the ComplexHeatmap (v2.6.2) package ${ }^{52}$. The clustering dendrogram in heatmaps were plotted using the Euclidean distance matrix.

\section{Pathway and functional enrichment analyses}

We used the PANTHER database (PantherDB v15, 16) ${ }^{53}$ to identify the enriched pathways related to our identified DMGs. In addition, to assess functional enrichment, we used the ReactomePA (v1.34.0) package ${ }^{54}$ with 1000 permutations and the BH-corrected $p$-values. Within the package, GO and Kyoto Encyclopedia of Genes and Genomes (KEGG) were used and using clusterProfiler ${ }^{55}$ (v3.18.1), we performed KEGG pathway ${ }^{56}$ enrichment analysis (data not shown). To enhance the visualization and better understanding of the enrichment result, GOplot ${ }^{57}$ (v1.0.2), another package was used. The pathway enrichment was also calculated using the topology-based ontology methods using RontoTools ${ }^{58}$ (v2.18.0), SPIA ${ }^{59}$ (v2.42.0) and pathview ${ }^{60}$ (v1.30.1) was used to visualize the related pathways with the KEGG pathway maps (data not shown).

\section{Venn and overlap analyses}

Venn analyses were performed to detect the DMGs overlapping between cell populations and between studies. We constructed the Venn diagrams by using matplotlib-venn package (https://github.com/konstantint/matplotlib-venn) using in-house python script. The overlap analyses were calculated and plotted using the go.Sunburst function from plotly using an in-house python script.

\section{Statistical analyses}

All differences with a $p$-value $<0.05$ were considered significant if not otherwise stated. We calculated family-wise error rate (FWER) using the BH correction method. All analyses were performed in R (v4.0.2) with the mentioned packages.

\section{Declarations}

\section{Data Availability}

The datasets generated and analysed during the current study are not publicly available due to Intellectual property rights but are available from the corresponding author on reasonable request.

\section{Acknowledgements}

This study was funded through generous grants from Forskningsrådet Sydöstra Sverige (FORSS932096), the Swedish Research Council (2015-02593 and 2018-02961) and the Swedish Heart Lung Foundation (20150709 and 20180613). J.D is a postdoctoral fellow supported through the Medical 
Infection and Inflammation Center (MIIC) at Linköping University. We direct our gratitude to the staff at Linköping University Hospital and the Vrinnevi Hospital in Norrköping for assistance in sample collection and all the subjects for donating samples. The DNA methylome data were generated at the Bioinformatics and Expression Analysis Core Facility at the Department of Biosciences and Nutrition, which is supported by the Board of Research at the Karolinska Institute, Stockholm. The computations were enabled by resources provided by the Swedish National Infrastructure for Computing (SNIC) at Linköping University campus partially funded by the Swedish Research Council through grant agreement no. 2018-05973.

\section{Author contributions}

M.L., N.I. and J.P. designed the study, I.P., N.I., C.B., and L.K., performed the laboratory work and the related analyses, J.D. and M.L. designed and performed the bioinformatic analyses of the data, J.D. and L.K wrote the scripts for analysis and created figures. N.I., I.P., J.D., L.K., C.B., and M.L wrote the manuscript. J.D and M.L are co-authors of a patent application "Biomarker for detection of mycobacterial infection and exposure" filed on February $2^{\text {nd }}$ with the Swedish Patent Registry (no. 100692).

\section{Additional information}

\section{Competing interests}

The authors declare no competing interests.

\section{References}

1. Fitchett, J. R., MacPherson, P. \& Corbett, E. L. Implementing the end TB strategy and the intersection with the sustainable development goals, 2016-2030. Transactions of the Royal Society of Tropical Medicine and Hygiene (2015) doi:10.1093/trstmh/trw010.

2. Donald, P. R. et al. Droplets, dust and Guinea pigs: An historical review of tuberculosis transmission research, 1878-1940. Int. J. Tuberc. Lung Dis. (2018) doi:10.5588/ijtld.18.0173.

3. Davis, J. M. \& Ramakrishnan, L. The Role of the Granuloma in Expansion and Dissemination of Early Tuberculous Infection. Cell (2009) doi:10.1016/j.cell.2008.11.014.

4. Verma, D. et al. Anti-mycobacterial activity correlates with altered DNA methylation pattern in immune cells from BCG-vaccinated subjects. Sci. Rep. 7, (2017).

5. Hasso-Agopsowicz, M., Scriba, T. J., Hanekom, W. A., Dockrell, H. M. \& Smith, S. G. Differential DNA methylation of potassium channel $\mathrm{KCa} 3.1$ and immune signalling pathways is associated with infant immune responses following BCG vaccination. Sci. Rep. (2018) doi:10.1038/s41598-01831537-9.

6. Kaufmann, E. et al. BCG Educates Hematopoietic Stem Cells to Generate Protective Innate Immunity against Tuberculosis. Cell (2018) doi:10.1016/j.cell.2017.12.031. 
7. Jonsson, J. et al. A borderline range for Quantiferon Gold In-Tube results. PLoS One (2017) doi:10.1371/journal.pone.0187313.

8. Sikkeland, L. I. B., Kongerud, J., Stangeland, A. M., Haug, T. \& Alexis, N. E. Macrophage enrichment from induced sputum [3]. Thorax (2007) doi:10.1136/thx.2006.073544.

9. Das, J., Verma, D., Gustafsson, M. \& Lerm, M. Identification of DNA methylation patterns predisposing for an efficient response to BCG vaccination in healthy BCG-naïve subjects. Epigenetics (2019) doi:10.1080/15592294.2019.1603963.

10. Ogryzko, N. V. et al. Hif-1a-Induced Expression of II-1 $\beta$ Protects against Mycobacterial Infection in Zebrafish. J. Immunol. 202, (2019).

11. Eklund, D. et al. Vitamin D enhances IL-1 $\beta$ secretion and restricts growth of Mycobacterium tuberculosis in macrophages from TB patients. Int. J. Mycobacteriology (2013) doi:10.1016/j.jijmyco.2012.11.001.

12. Liu, P. T. et al. Toll-like receptor triggering of a vitamin D-mediated human antimicrobial response. Science (80-.). 311, 1770-1773 (2006).

13. Hölscher, C. et al. Chemical p38 MAP kinase inhibition constrains tissue inflammation and improves antibiotic activity in Mycobacterium tuberculosis-infected mice. Sci. Rep. (2020) doi:10.1038/s41598-020-70184-x.

14. Brandenburg, J. \& Reiling, N. The Wnt blows: On the functional role of Wnt signaling in mycobacterium tuberculosis infection and beyond. Frontiers in Immunology (2016) doi:10.3389/fimmu.2016.00635.

15. Castro, R. C. et al. NOTCH1 and DLL4 are involved in the human tuberculosis progression and immune response activation. Tuberculosis (2020) doi:10.1016/j.tube.2020.101980.

16. Eklund, D. et al. Human gene variants linked to enhanced NLRP3 activity limit intramacrophage growth of mycobacterium tuberculosis. in Journal of Infectious Diseases (2014). doi:10.1093/infdis/jit572.

17. Raffetseder, J. et al. Replication rates of mycobacterium tuberculosis in human macrophages do not correlate with mycobacterial antibiotic susceptibility. PLoS One (2014) doi:10.1371/journal.pone.0112426.

18. Kaufmann, E. et al. BCG Educates Hematopoietic Stem Cells to Generate Protective Innate Immunity against Tuberculosis. Cell 172, 176-190.e19 (2018).

19. Saeed, S. et al. Epigenetic programming of monocyte-to-macrophage differentiation and trained innate immunity. Science (80-). (2014) doi:10.1126/science.1251086.

20. Mourits, V. P. et al. BCG-Induced Trained Immunity in Healthy Individuals: The Effect of Plasma Muramyl Dipeptide Concentrations. J. Immunol. Res. (2020) doi:10.1155/2020/5812743.

21. Kleinnijenhuis, J. et al. Bacille Calmette-Guérin induces NOD2-dependent nonspecific protection from reinfection via epigenetic reprogramming of monocytes. Proc. Natl. Acad. Sci. U. S. A. (2012) doi:10.1073/pnas.1202870109. 
22. Russell, D. G., Cardona, P. J., Kim, M. J., Allain, S. \& Altare, F. Foamy macrophages and the progression of the human tuberculosis granuloma. Nature Immunology (2009) doi:10.1038/ni.1781.

23. Benn, C. S., Fisker, A. B., Whittle, H. C. \& Aaby, P. Revaccination with Live Attenuated Vaccines Confer Additional Beneficial Nonspecific Effects on Overall Survival: A Review. EBioMedicine (2016) doi:10.1016/j.ebiom.2016.07.016.

24. Thysen, S. M. et al. Neonatal BCG vaccination and child survival in TB-exposed and TB-unexposed children: A prospective cohort study. BMJ Open (2020) doi:10.1136/bmjopen-2019-035595.

25. Verrall, A. J. et al. Early Clearance of Mycobacterium tuberculosis: The INFECT Case Contact Cohort Study in Indonesia. J. Infect. Dis. (2019) doi:10.1093/infdis/jiz168.

26. Arts, R. J. W., Joosten, L. A. B. \& Netea, M. G. Immunometabolic circuits in trained immunity. Seminars in Immunology (2016) doi:10.1016/j.smim.2016.09.002.

27. Kumar, R. et al. Immunometabolism of Phagocytes During Mycobacterium tuberculosis Infection. Frontiers in Molecular Biosciences (2019) doi:10.3389/fmolb.2019.00105.

28. Kleinnijenhuis, J. et al. Bacille Calmette-Guerin induces NOD2-dependent nonspecific protection from reinfection via epigenetic reprogramming of monocytes. Proc. Natl. Acad. Sci. 109, 17537-17542 (2012).

29. Harding, J. S. et al. VEGF-A from Granuloma Macrophages Regulates Granulomatous Inflammation by a Non-angiogenic Pathway during Mycobacterial Infection. Cell Rep. (2019) doi:10.1016/j.celrep.2019.04.072.

30. Rao Muvva, J., Parasa, V. R., Lerm, M., Svensson, M. \& Brighenti, S. Polarization of Human MonocyteDerived Cells With Vitamin D Promotes Control of Mycobacterium tuberculosis Infection. Front. Immunol. (2020) doi:10.3389/fimmu.2019.03157.

31. Cox, M. A. et al. Beyond neurotransmission: acetylcholine in immunity and inflammation. Journal of Internal Medicine (2020) doi:10.1111/joim.13006.

32. Cox, M. A. et al. Choline acetyltransferase-expressing $\mathrm{T}$ cells are required to control chronic viral infection. Science (80-.). (2019) doi:10.1126/science.aau9072.

33. Ganor, Y. \& Levite, M. The neurotransmitter glutamate and human T cells: Glutamate receptors and glutamate-induced direct and potent effects on normal human T cells, cancerous human leukemia and lymphoma T cells, and autoimmune human T cells. J. Neural Transm. (2014) doi:10.1007/s00702-014-1167-5.

34. Ganor, Y. \& Levite, M. The neurotransmitter glutamate and human T cells: Glutamate receptors and glutamate-induced direct and potent effects on normal human T cells, cancerous human leukemia and lymphoma T cells, and autoimmune human T cells. J. Neural Transm. 121, 983-1006 (2014).

35. Kuhn, J. H., Li, W., Radoshitzky, S. R., Choe, H. \& Farzan, M. Severe acute respiratory syndrome coronavirus entry as a target of antiviral therapies. Antiviral Therapy (2007).

36. Lake, M. A., Ambrose, L. R., Lipman, M. C. I. \& Lowe, D. M. ' 'Why me, why now?' Using clinical immunology and epidemiology to explain who gets nontuberculous mycobacterial infection. BMC Med. (2016) doi:10.1186/s12916-016-0606-6. 
37. Das, J., Idh, N., Sikkeland, L. I. B., Paues, J. \& Lerm, M. DNA methylome-based validation of induced sputum as an effective protocol to study lung immunity: construction of a classifier of pulmonary cell types. bioRxiv (2021) doi:10.1101/2021.03.12.435086.

38. Ugarte-Gil, C., Elkington, P. T., Gotuzzo, E., Friedland, J. S. \& Moore, D. A. J. Induced sputum is safe and well-tolerated for TB diagnosis in a resource-poor primary healthcare setting. American Journal of Tropical Medicine and Hygiene (2015) doi:10.4269/ajtmh.14-0583.

39. Alexis, N., Soukup, J., Ghio, A. \& Becker, S. Sputum phagocytes from healthy individuals are functional and activated: A flow cytometric comparison with cells in bronchoalveolar lavage and peripheral blood. Clin. Immunol. (2000) doi:10.1006/clim.2000.4911.

40. Alexis, N. E. et al. In vivo particle uptake by airway macrophages in healthy volunteers. Am. J. Respir. Cell Mol. Biol. (2006) doi:10.1165/rcmb.2005-03730C.

41. Rueden, C. T. et al. ImageJ2: ImageJ for the next generation of scientific image data. BMC Bioinformatics (2017) doi:10.1186/s12859-017-1934-z.

42. Schindelin, J. et al. Fiji: An open-source platform for biological-image analysis. Nature Methods (2012) doi:10.1038/nmeth.2019.

43. Maksimovic, J., Gordon, L. \& Oshlack, A. SWAN: Subset-quantile within array normalization for illumina infinium HumanMethylation450 BeadChips. Genome Biol. (2012) doi:10.1186/gb-2012-136-r44.

44. Aryee, M. J. et al. Minfi: A flexible and comprehensive Bioconductor package for the analysis of Infinium DNA methylation microarrays. Bioinformatics (2014) doi:10.1093/bioinformatics/btu049.

45. Teschendorff, A. E. et al. A beta-mixture quantile normalization method for correcting probe design bias in Illumina Infinium 450 k DNA methylation data. Bioinformatics (2013) doi:10.1093/bioinformatics/bts680.

46. Morris, T. J. et al. ChAMP: 450k Chip Analysis Methylation Pipeline. Bioinformatics 30, 428-430 (2014).

47. Houseman, E. A., Molitor, J. \& Marsit, C. J. Reference-free cell mixture adjustments in analysis of DNA methylation data. Bioinformatics (2014) doi:10.1093/bioinformatics/btu029.

48. Ritchie, M. et al. limma powers differential expression analyses for RNA-sequencing and microarray studies | Nucleic Acids Research | Oxford Academic. limma powers Differ. Expr. Anal. RNAsequencing microarray Stud. (2015).

49. Andrews S. FastQC A Quality control tool for high throughput sequence data. Babraham Bioinfo (2018).

50. Langmead, B. \& Salzberg, S. L. Fast gapped-read alignment with Bowtie 2. Nat. Methods (2012) doi:10.1038/nmeth.1923.

51. Krueger, F. \& Andrews, S. R. Bismark: A flexible aligner and methylation caller for Bisulfite-Seq applications. Bioinformatics (2011) doi:10.1093/bioinformatics/btr167. 
52. Li, H. et al. The Sequence Alignment/Map format and SAMtools. Bioinformatics (2009) doi:10.1093/bioinformatics/btp352.

53. Gaspar, J. M. \& Hart, R. P. DMRfinder: Efficiently identifying differentially methylated regions from MethylC-seq data. BMC Bioinformatics (2017) doi:10.1186/s12859-017-1909-0.

54. Akalin, A. et al. MethylKit: a comprehensive R package for the analysis of genome-wide DNA methylation profiles. Genome Biol. (2012) doi:10.1186/gb-2012-13-10-R87.

55. Galili, T. dendextend: An R package for visualizing, adjusting and comparing trees of hierarchical clustering. Bioinformatics (2015) doi:10.1093/bioinformatics/btv428.

56. Paradis, E. \& Schliep, K. Ape 5.0: An environment for modern phylogenetics and evolutionary analyses in R. Bioinformatics (2019) doi:10.1093/bioinformatics/bty633.

57. Blighe, K, S Rana, and M. L. EnhancedVolcano: Publication-ready volcano plots with enhanced colouring and labeling. (2018).

58. Anand, L. \& Rodriguez Lopez, C. M. ChromoMap: An R package for Interactive Visualization and Annotation of Chromosomes. bioRxiv (2019) doi:10.1101/605600.

59. Gu, Z., Eils, R. \& Schlesner, M. Complex heatmaps reveal patterns and correlations in multidimensional genomic data. Bioinformatics (2016) doi:10.1093/bioinformatics/btw313.

60. Thomas, P. D. et al. PANTHER: A library of protein families and subfamilies indexed by function. Genome Res. 13, 2129-2141 (2003).

61. Yu, G. \& He, Q. Y. ReactomePA: An R/Bioconductor package for reactome pathway analysis and visualization. Mol. Biosyst. (2016) doi:10.1039/c5mb00663e.

62. Yu, G., Wang, L. G., Han, Y. \& He, Q. Y. ClusterProfiler: An R package for comparing biological themes among gene clusters. Omi. A J. Integr. Biol. (2012) doi:10.1089/omi.2011.0118.

63. Kanehisa, M. KEGG: Kyoto Encyclopedia of Genes and Genomes. Nucleic Acids Res. (2000) doi:10.1093/nar/28.1.27.

64. Walter, W., Sánchez-Cabo, F. \& Ricote, M. GOplot: An R package for visually combining expression data with functional analysis. Bioinformatics (2015) doi:10.1093/bioinformatics/btv300.

65. Luo, W., Friedman, M. S., Shedden, K., Hankenson, K. D. \& Woolf, P. J. GAGE: Generally applicable gene set enrichment for pathway analysis. BMC Bioinformatics (2009) doi:10.1186/1471-2105-10161.

66. Voichita, C., Ansari, S. \& Draghici, S. ROntoTools: The R Onto-Tools suite. https://bioconductor.riken.jp/packages/3.10/bioc/vignettes/ROntoTools/inst/doc/rontotools.pdf (2019).

67. Tarca, A. L. et al. A novel signaling pathway impact analysis. Bioinformatics (2009) doi:10.1093/bioinformatics/btn577.

68. Luo, W. \& Brouwer, C. Pathview: an R/Bioconductor package for pathway-based data integration and visualization. Bioinformatics 29, 1830-1831 (2013). 


\section{Tables}

Table 1. Demographic data of the participants in the first and second recruitment.

${ }^{\dagger}$ The standard deviation of the mean values is added to the age, height, weight and BMI.

* Borderline-positive.

Characteristics

Mean age (year)

Mean height $(\mathrm{cm})^{\dagger}$

Mean weight $(\mathrm{kg})^{\dagger}$

Mean Body Mass Index $(\mathrm{BMI})^{\dagger}$

Sex (male/female)

Smoking (current/previous/never)

BCG (yes/no)

IGRA-positive/IGRA-negative

TB patient/contact
TB-exposed $(\mathrm{n}=$

4)

$36 \pm 12$

$173 \pm 4$

$69 \pm 8$

$23 \pm 3$

$1 / 3$

$0 / 1 / 3$

$3 / 1$

$2 / 2$

$1 / 3$
Controls $(\mathrm{n}=$ 6)

$27 \pm 6$

$176 \pm 7$

$77 \pm 16$

$24 \pm 5$

$4 / 2$

$0 / 0 / 6$

$6 / 0$

$1 * / 5$

$0 / 0$

$1 / 2$

Figures 


\section{First enrollment}

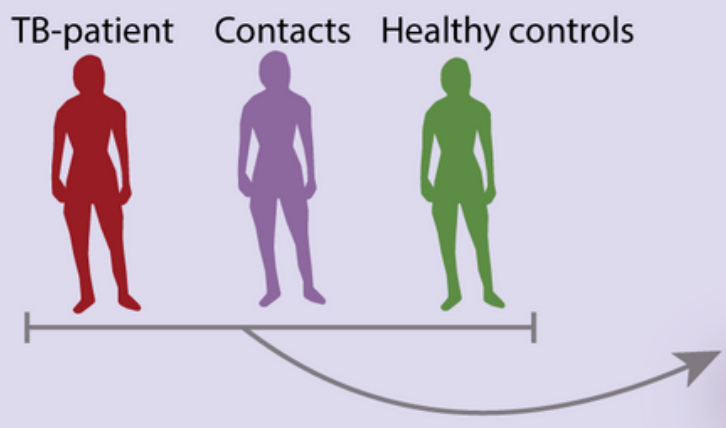

\section{Second enrollment}

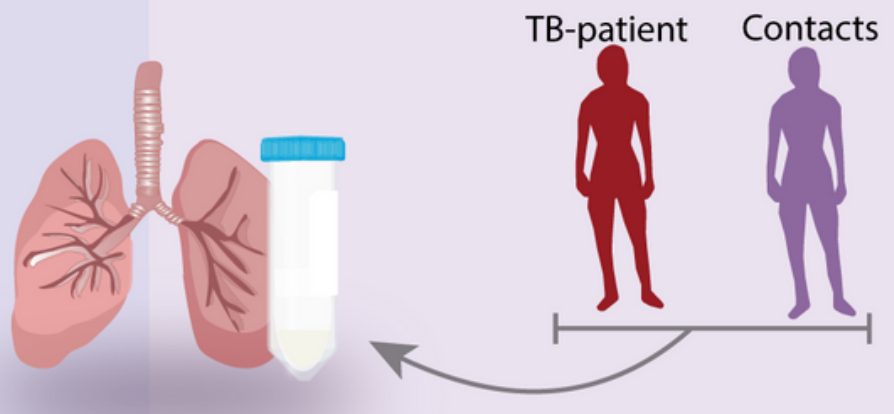

Sputum induction

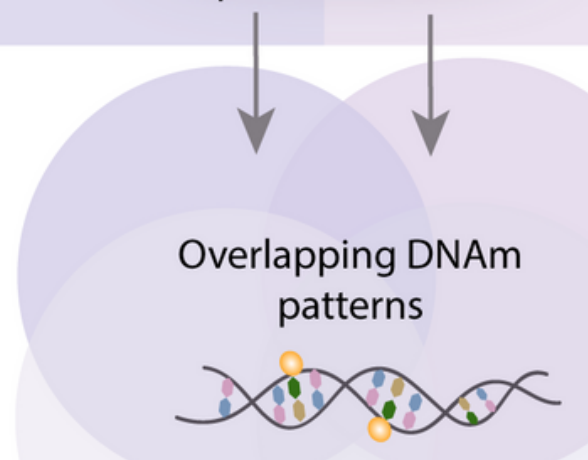

3. In vitro experiment

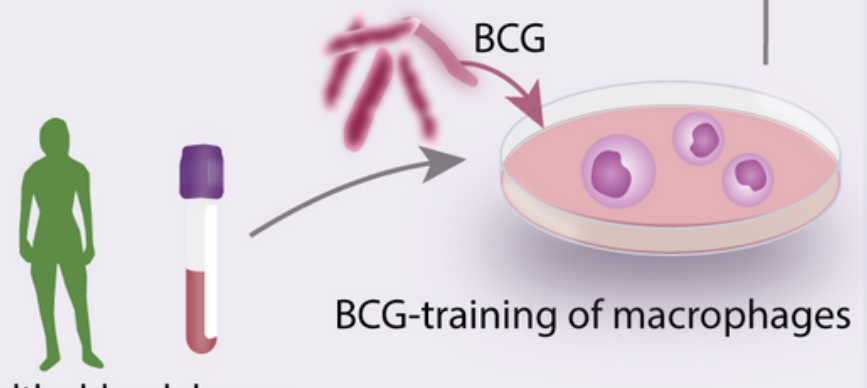

Healthy blood donors

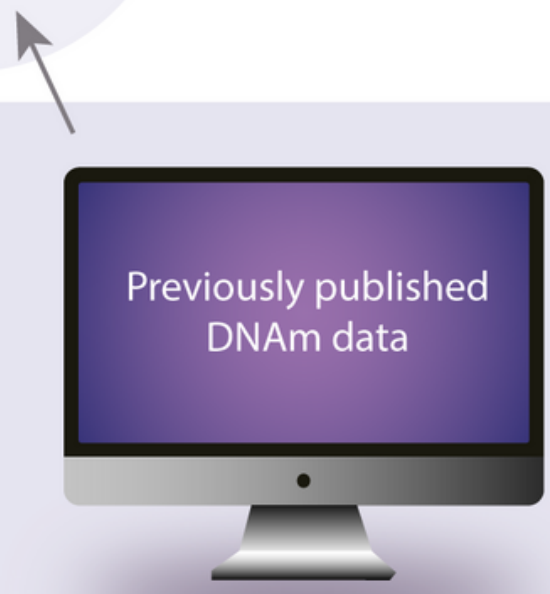

\section{Figure 1}

Schematic overview of the project workflow. Sputum and blood were isolated from TB-exposed individuals and controls and the DNA methylomes were analyzed for the different cell types. Macrophages were isolated from healthy blood donors and exposed to the Bacillus Calmette-Guérin (BCG) vaccine in vitro. The BCG-exposed macrophages were subjected to DNA methylome analysis. TB, tuberculosis; DNAm, DNA-methylation; BCG, Bacillus Calmette-Guérin. 
a

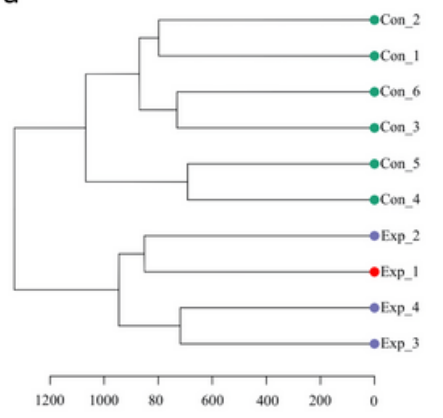

b

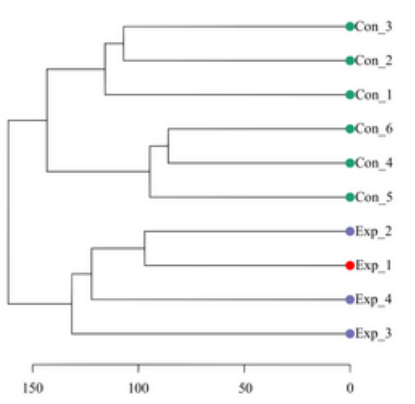

d

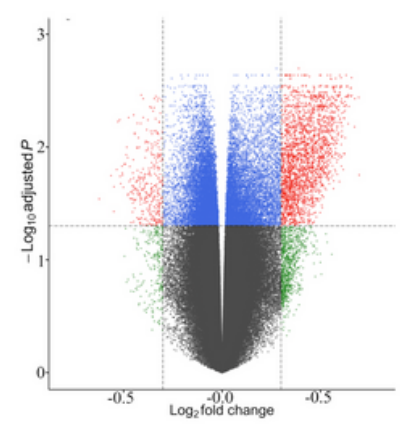

f

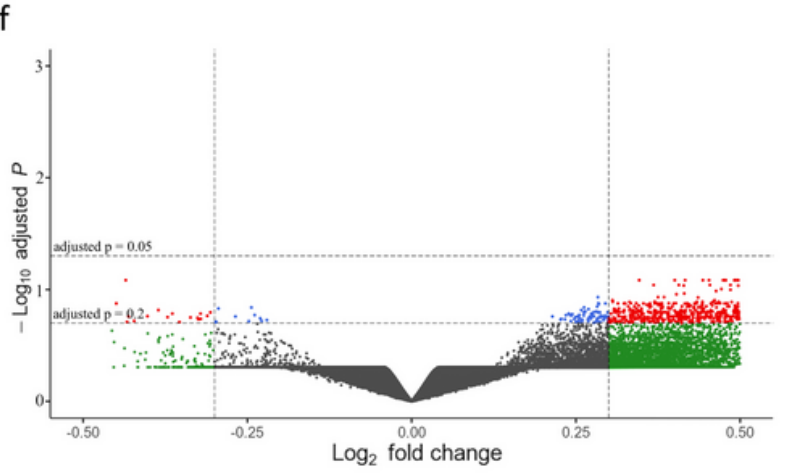

e

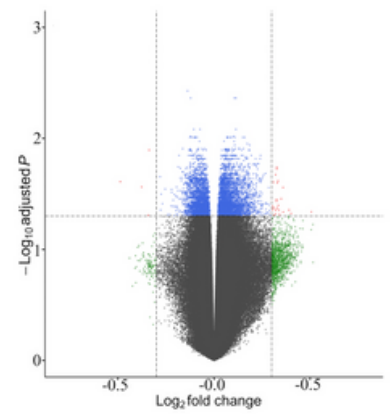

Figure 2

C

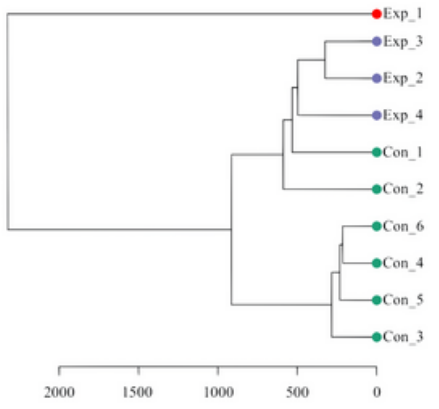

$$
\text { ( }
$$




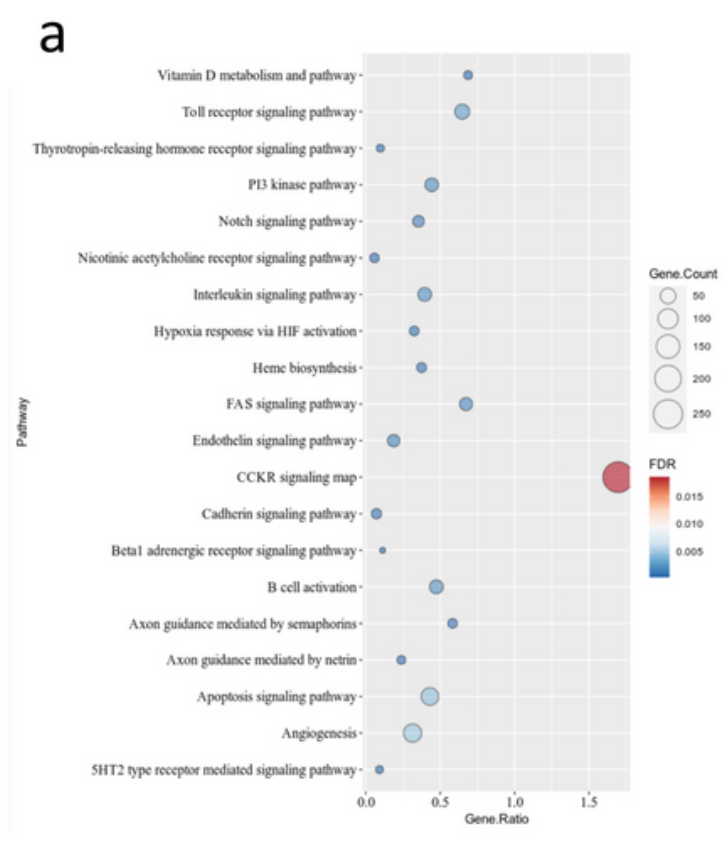

b
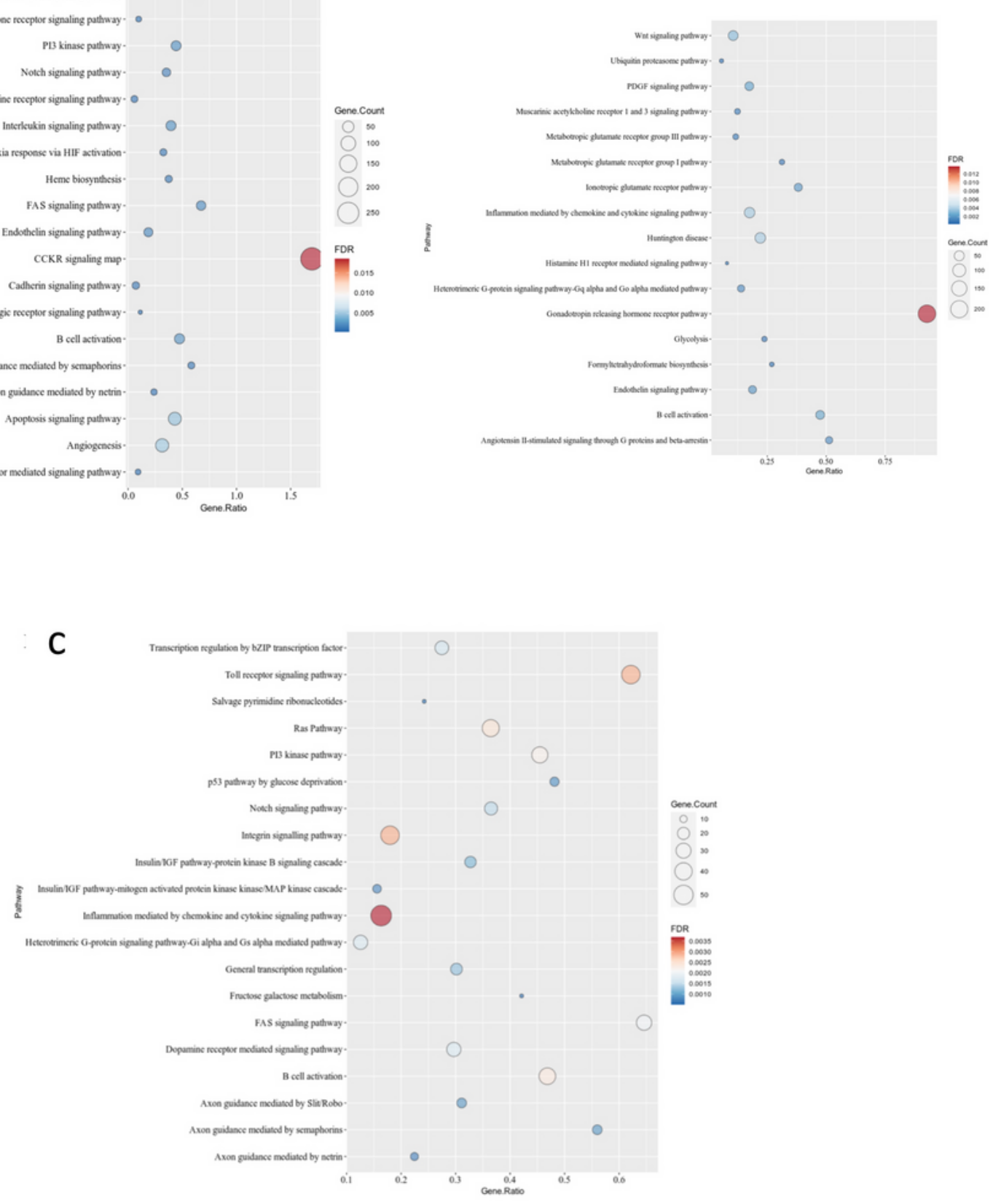

\section{Figure 3}

PANTHER pathway analysis of the identified DMGs with the cut-offs for the different cell populations given in Figure 2. Dot plots show the gene ratio, gene counts and FDR-corrected $p$-value for a. HLA-DR (top 20 pathways), b. CD3 (total 17 pathways). c. PBMC (top 20 pathways). 
a

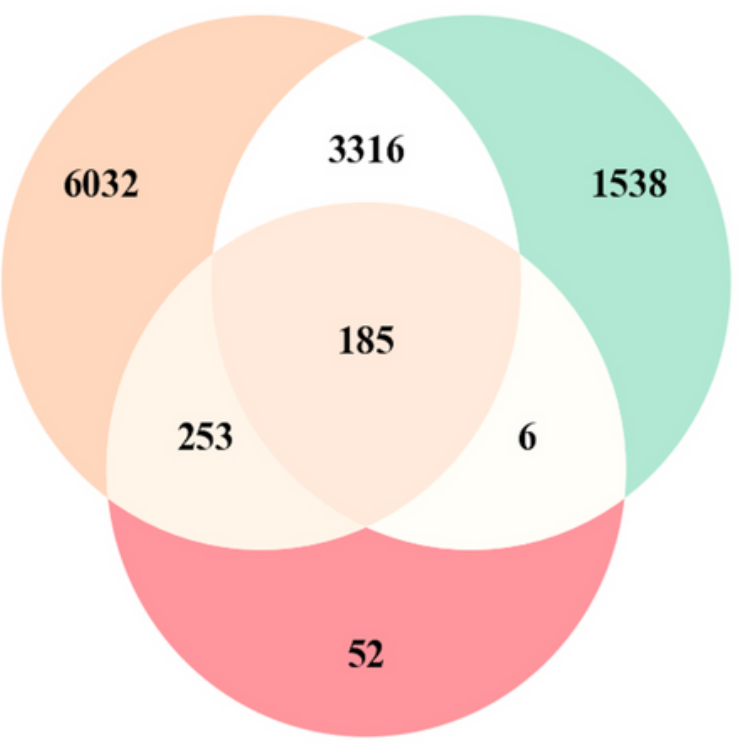

b

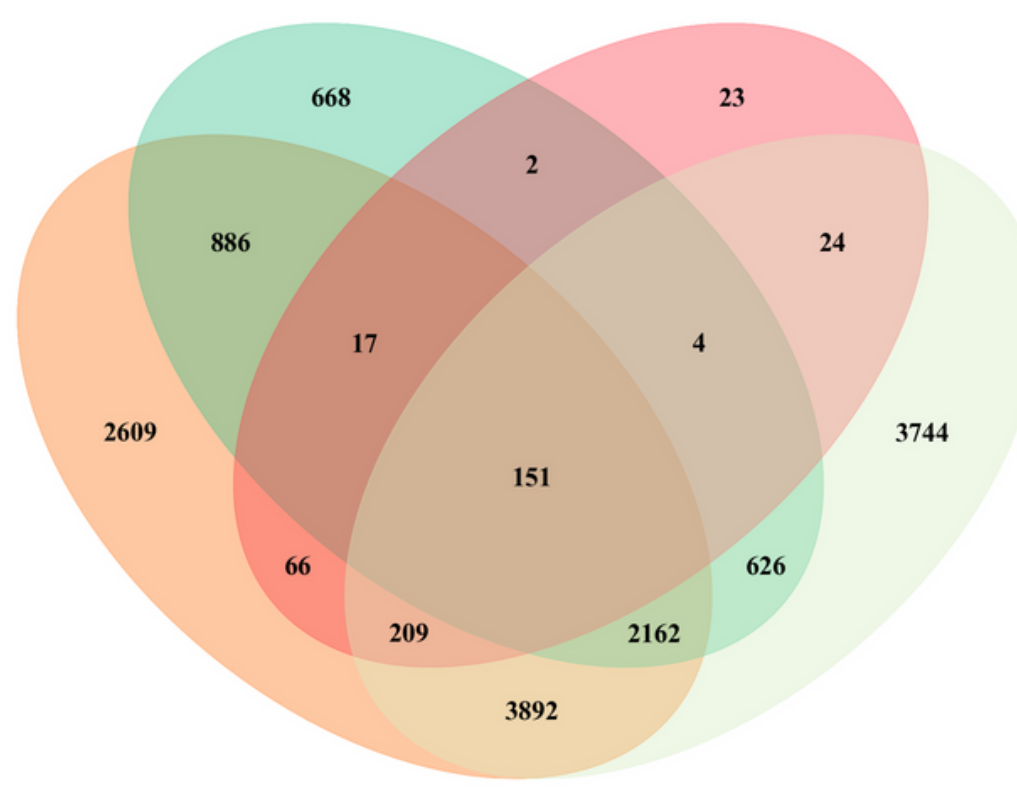

\section{Figure 4}

Venn analyses comparing DMGs, pathways and GO terms between different datasets. a. Overlapping DMGs derived from the HLA-DR (orange), CD3 (green) and PBMC (red) DNA methylomes. b. Overlapping DMGs from this study and from our previous work on BCG induced DMGs (light green). 
a

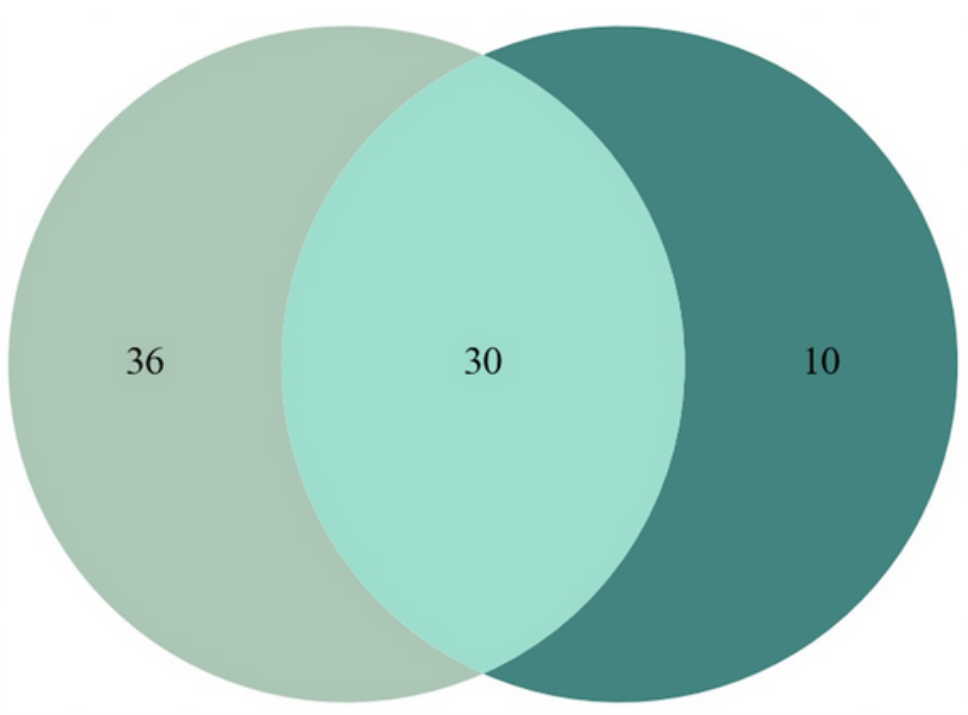

b

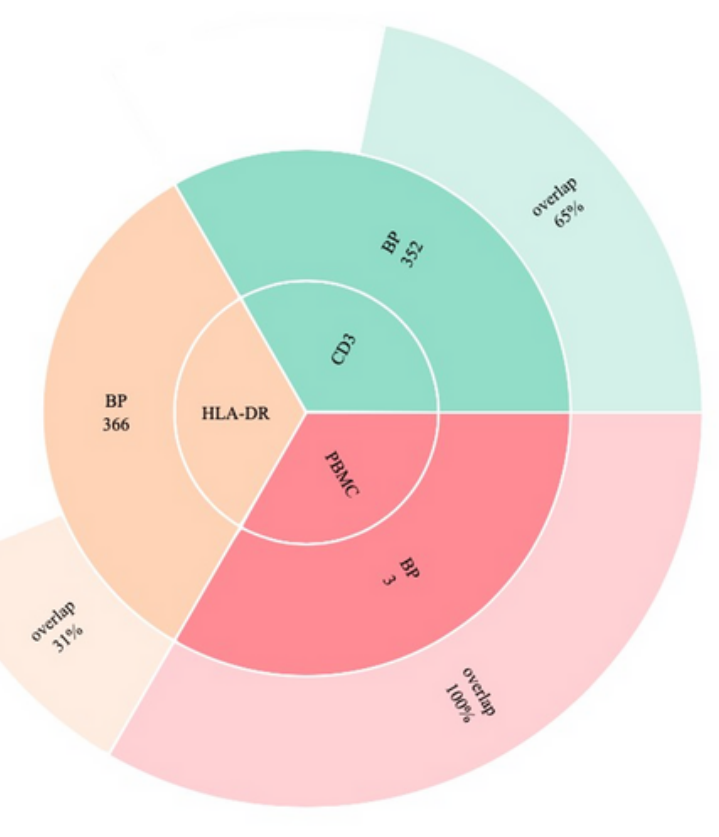

C

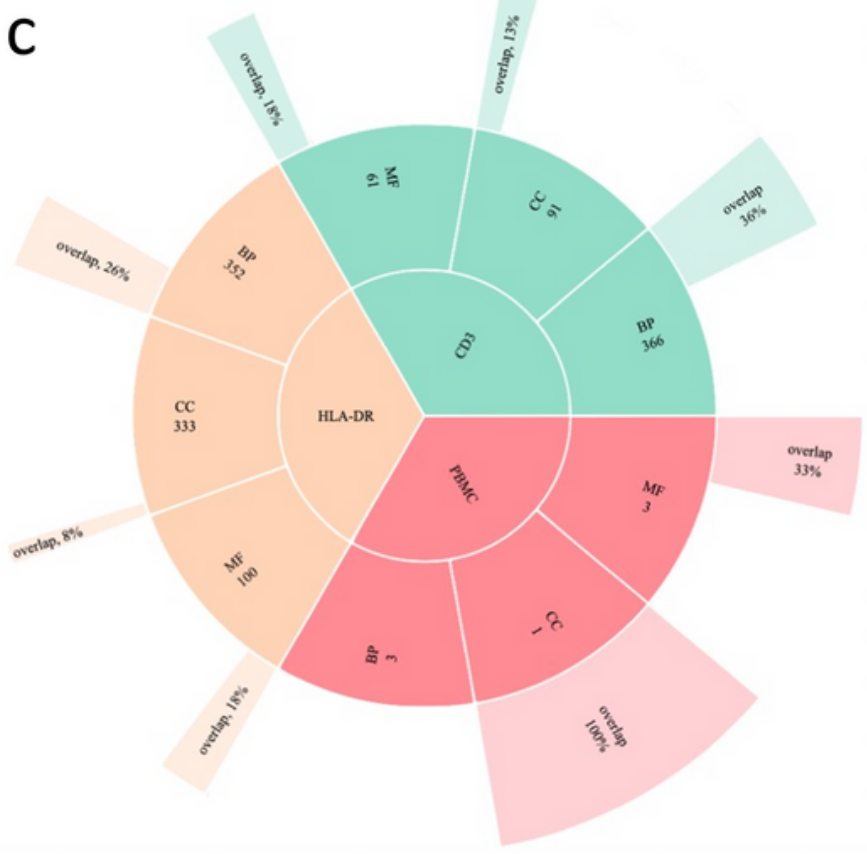

\section{Figure 5}

Pathway overlap with other studies' results. a. Venn diagram describing the number of Panther pathways overlapping between the ones derived from the 185 common DMGs in this study (dark green) and HassoAgopsowicz et al. (human BCG vaccine study, light green) a. Sunburst Plot describing the overlap of enriched $\mathrm{GO}$ biological processes emerging from a comparison between the $\mathrm{GO}$ data derived from the 185 common DMGs (Figure 4a) and Kaufmann et al (BCG study performed in mouse PBMCs). c. 
Sunburst Plot describing the overlap of enriched $\mathrm{GO}$ biological processes emerging from a comparison between the GO data derived from the 185 common DMGs and Saeed et al (study on trained immunity induced by b-glucan).

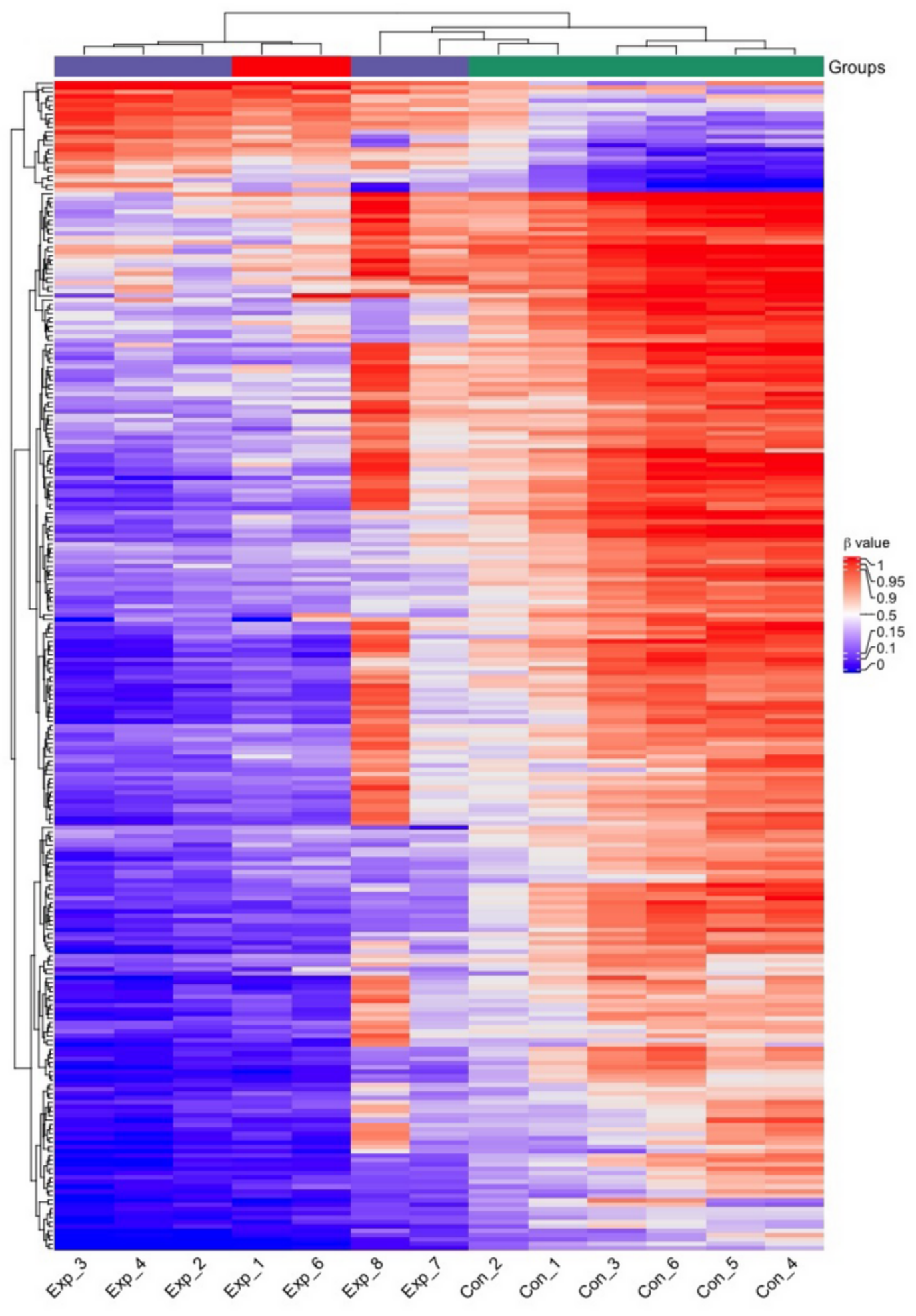

Figure 6 
Heatmap of the HLA-DR-derived $\beta$ values of the signature's 284 CpG sites of the 6 initial subjects (Exp_1-4 and Con_1-6) and the three additional exposed subjects (Exp_6-8). Purple=exposed, red=TB index case, green=controls.
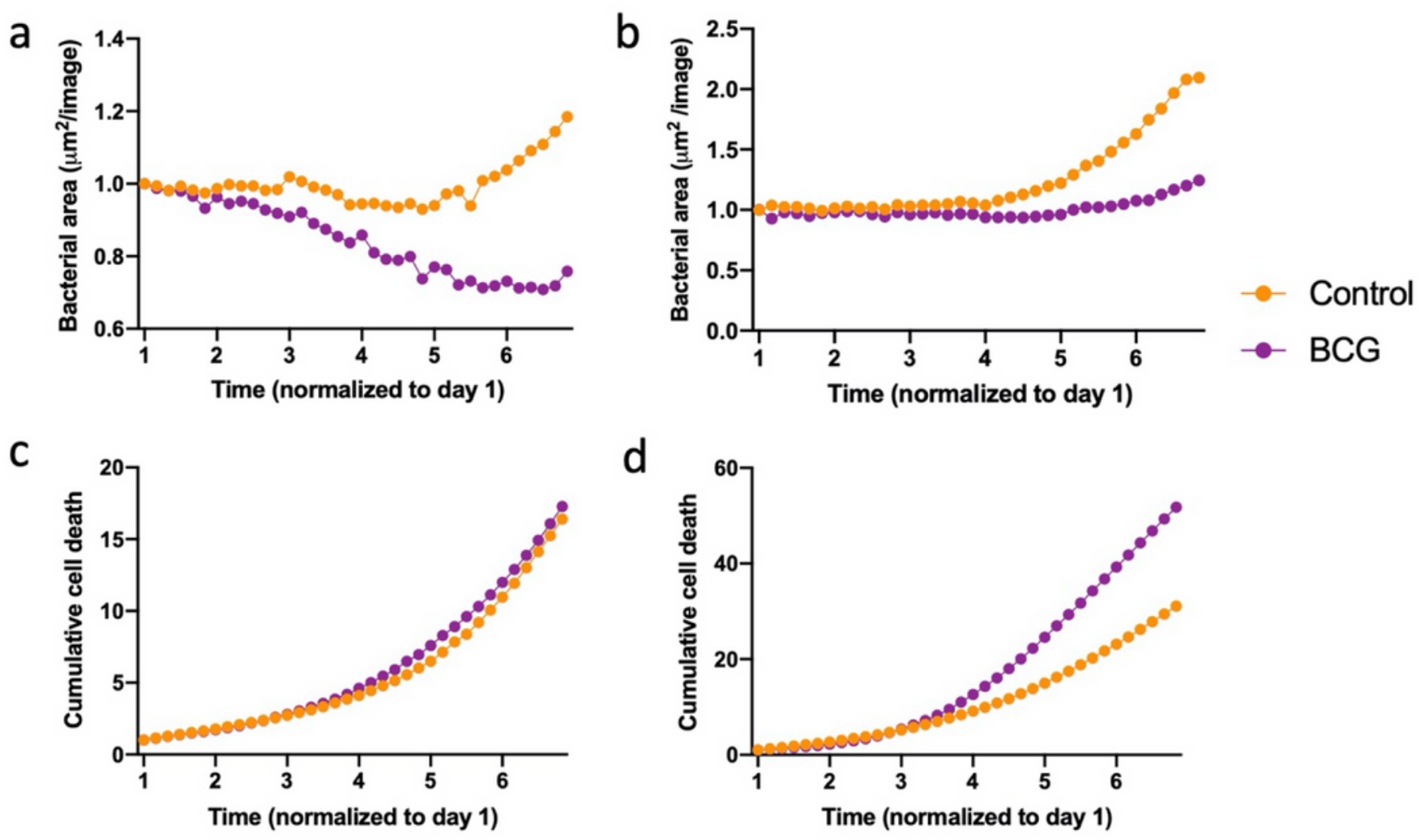

e

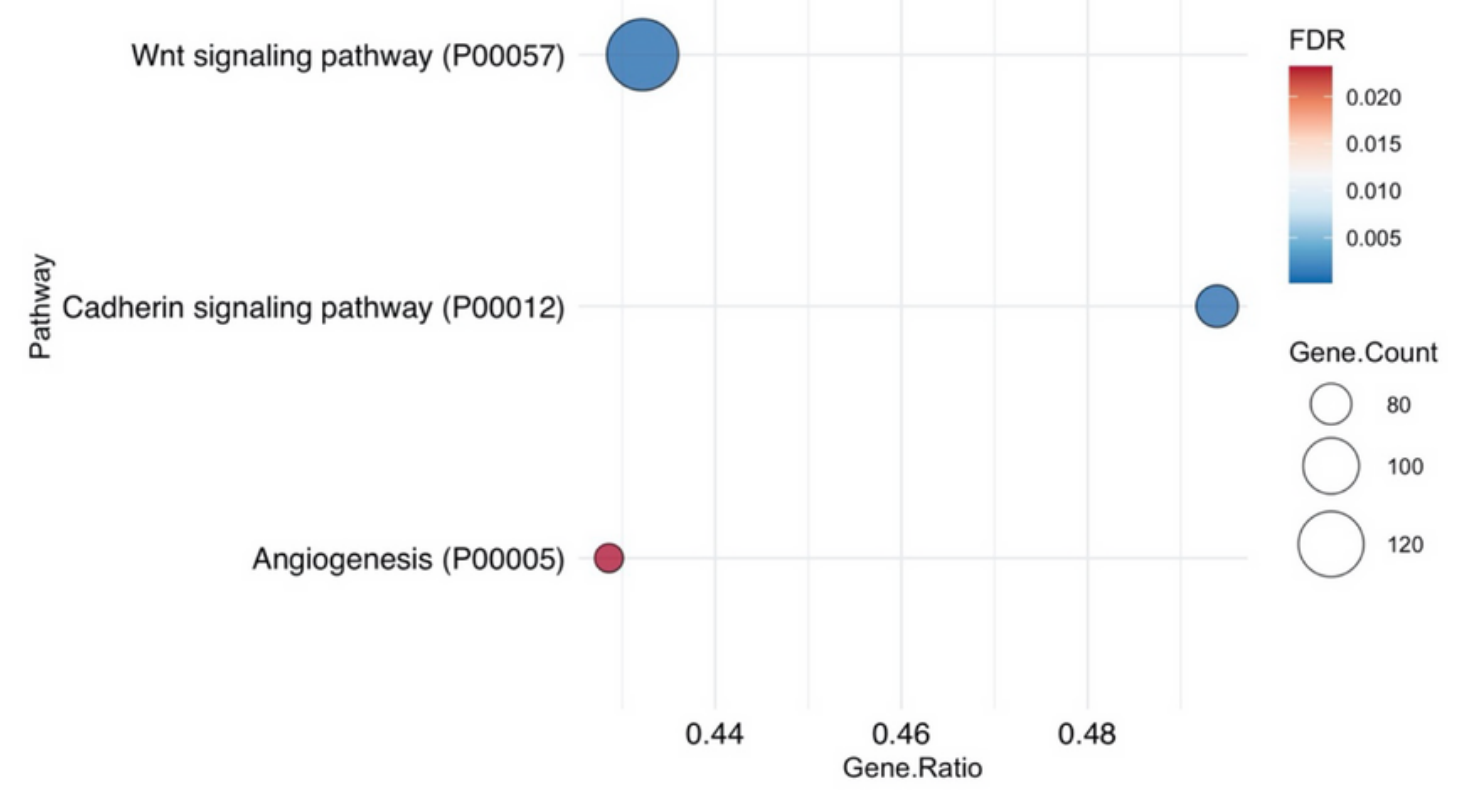

Figure 7 
Macrophages were BCG-trained (purple) or left untreated (orange) before infection with $M$. tuberculosis (H37rv-GFP) or DNAm analysis. a-b. Total bacterial area ( $\mu \mathrm{m}^{2} /$ image) measured by green fluorescence over time. c-d. Cumulative cell death measured by the red fluorescent DRAQ7 DNA stain marking the nuclei of non-viable cells. a-d shows data from one donor separately with the median of two replicates per timepoint.e. PANTHER pathway analysis of the identified DMGs from DNA methylation analysis in the human primary macrophages exposed to BCG compared to controls. Dot plots show the gene ratio, gene counts and FDR-corrected $p$-value for 3 significantly over-represented pathways.

\section{Supplementary Files}

This is a list of supplementary files associated with this preprint. Click to download.

- SupplementaryInformation.docx 